\title{
BMJ Open Robotic versus open urological oncological surgery: study protocol of a systematic review and meta-analysis
}

\author{
Giovanni E Cacciamani (D), Karanvir Gill, Inderbir S Gill
}

To cite: Cacciamani GE, Gill K, Gill IS. Robotic versus open urological oncological surgery: study protocol of a systematic review and meta-analysis. BMJ Open 2020;10:e036609. doi:10.1136/ bmjopen-2019-036609

- Prepublication history and additional material for this paper are available online. To view these files, please visit the journal online (http://dx.doi. org/10.1136/bmjopen-2019036609).

Received 23 December 2019 Revised 31 December 2019 Accepted 08 January 2020

Check for updates

(c) Author(s) (or their employer(s)) 2020. Re-use permitted under CC BY-NC. No commercial re-use. See rights and permissions. Published by BMJ.

USC Institute of Urology and Catherine and Joseph Aresty Department of Urology, University of Southern California, Los Angeles, California, USA

\section{Correspondence to} Dr Giovanni E Cacciamani; giovanni.cacciamani@gmail. com

\begin{abstract}
Introduction Minimally invasive surgery in urology has grown considerably in application since its initial description in the early 1990s. Herein, we present the protocol for a systematic review and meta-analysis comparing open versus robotic urological oncological surgery for various clinically relevant outcomes, as well as to assess their comparative penetrance over the past 20 years (2000-2020).
\end{abstract}

Methods and analysis We will document the penetrance of robotic versus open surgery in the urological oncological field using a national database.

Second, we will perform a systematic review and metaanalysis of all published full-text English and non-English language articles from Pubmed, Scopus and Web of Science search engines on surgical treatment of localised prostate, bladder, kidney and testis cancer published between 1 st January 2000 to 10th January 2020. We will focus on the highest-volume urological oncological surgeries, namely, radical prostatectomy, radical cystectomy, partial nephrectomy, radical nephrectomy and retroperitoneal lymph node dissection. Study inclusion criteria will comprise clinical trials and prospective and retrospective studies (cohort or case-control series) comparing robotic versus open surgery. Exclusion criteria will comprise meta-analyses, multiple papers with overalapping study-periods, studies analysing national databases and case series describing only one approach (robotic or open). Risk of bias for included studies will be assessed by the appropriate Cochrane risk of bias tool. Principal outcomes assessed will include perioperative, functional, oncological survival and financial outcomes of open versus robotic uro-oncological surgery. Sensitivity analyses will be performed to correlate outcomes of interest with key baseline characteristics and surrogates of surgical expertise.

Ethics and dissemination This comprehensive systematic review and meta-analysis will provide rigorous, consolidated information on contemporary outcomes and trends of open versus robotic urological oncological surgery based on all the available literature. These aggregate data will help physicians better advise patients seeking surgical care for urological cancers. PROSPERO registration number CRD42017064958.

\section{INTRODUCTION}

Minimally invasive surgery (MIS) in urology has grown considerably in application since

\section{Strengths and limitations of this study}

The strengths of this protocol include standardised and transparent methods and processes that minimise possible biases. The quality of evidence will be assessed to provide confidence in the effect estimates, thereby reporting the strength of recommendations and deriving clinical meaning from the statistical findings.

- The present study will be the first to evaluate the five highest-volume open versus robotic urological oncological surgeries over 20 years (2000-2020) as regards outcomes, costs and comparative penetrance.

- This meta-analysis will be used to inform future studies to fulfil gaps in knowledge in comparative outcomes of open and robotic urological oncological surgery.

- This protocol is the first to correlate outcomes of interest with baseline characteristics and surrogates of surgical expertise (ie, surgical case volumes and year of publication)

- Limitations of the study will be the paucity of randomised controlled trials and the heterogeneity among available publications.

its initial description over three decades ago. Initially, throughout the 1990s, urological MIS consisted mostly of laparoscopic surgery, which, due to its skill-intensive nature, was slow to be adopted by practitioners in the field. Robotic surgery, initially applied to urology in the early 2000s, has gradually, and now virtually completely, replaced laparoscopy in uro-oncological surgery in the USA. Specifically, robotics has now also emerged as a viable alternative to open surgery in many uro-oncological applications. This shift away from open surgery and towards robotic surgery is relatively recent and significant.

Robotic surgery is increasing in application and scope. By the end of 2017, a total of 4409 robotic platforms had been installed globally, 43000 robotic surgeons trained, over 5 million robotic surgeries performed across various disciplines worldwide, with over 15 
000 publications. ${ }^{1}$ In 2017, total revenue for Intuitive Surgical, the sole manufacturer of the da Vinci robot was reported US $\$ 3.1$ billion. Globally, estimated annual case volumes increased from 136000 in 2008 to 877000 in 2017; in urology, robotic procedures increased from 85 000 to 118000 annually (2010-2017). ${ }^{2}$ Given the significant increase in the number of robotic surgeries for prostate, bladder and kidney cancer, it is now a major domain in urological oncological surgery.

We seek to examine the state-of-the-field of open versus robotic urological oncological surgery over the past 20 years (2000-2020) by assessing the highest-volume oncological surgeries: radical prostatectomy (RP) for prostate cancer, radical cystectomy (RC) for bladder cancer, partial nephrectomy (PN) and radical nephrectomy (RN) for kidney cancer and retroperitoneal lymph node dissection (RPLND) for testis cancer. For each of these procedures that have the requisite published comparative data, we will compare the two surgical approaches, open versus robotic, as follows: for prostate cancer-open radical prostatectomy (ORP) versus robotic radical prostatectomy (RRP); for bladder cancer-open radical cystectomy (ORC) versus robotic radical cystectomy (RRC); for kidney cancer-open partial nephrectomy (OPN) versus robotic partial nephrectomy (RPN) and open radical nephrectomy (ORN) versus robotic radical nephrectomy (RRN); and for testis cancer-open retroperitoneal lymph node dissection (ORPLND) versus robotic retroperitoneal lymph node dissection (RRPLND). For each procedure type, we will interrogate a national database (the Premier Database) to assess comparative penetrance in the field; and we will compare open versus robotic surgery as regards perioperative data, procedural morbidity, oncological outcomes, functional outcomes and financial costs. This penetrance analysis, systematic review and meta-analysis will help inform physicians and patients about contemporary trends in oncological surgery for urological cancers.

\section{OBJECTIVES}

The aim of this study is to present a protocol paper for a rigorous systematic review and meta-analysis of all published comparative studies of robotic versus open urological oncological surgery from 2000 to 2020, as well as an evaluation of the Premier Database, to answer the following key questions (KQs).

\section{Key Questions}

KQ 1: What is the annual comparative penetrance of open vers robotic urological oncological surgery?

KQ2: What are the perioperative outcomes and complications of open versus robotic urological oncological surgery?

KQ3: What are the oncological outcomes and shortterm survival data of open versus robotic urological oncological surgery?
KQ4: What are the functional outcomes and healthrelated quality-of-life outcomes of open versus robotic urological oncological surgery?

KQ5: What is the financial cost comparison of open vers robotic urological oncological surgery?

For each procedure type, we will methodically and separately compare open vs robotic surgery as regards five key questions: penetrance in the field, perioperative data, procedural morbidity, oncological outcomes, functional outcomes and financial cost

\section{METHODS}

\section{Trends analysis: penetrance in the-field}

We will analyse data from the Premier Hospital Database (Premier Inc, Charlotte, North Carolina, USA), a nationally representative all-payer database capturing more than 152 million hospital inpatient discharges in 700 hospitals, representative of about $20 \%$ of all inpatient admissions in the USA. The Premier Database has been validated and used in previous studies. ${ }^{3-7}$

Using the International Classification of Diseases, ninth Revision codes, we will identify patients diagnosed with prostate cancer (code 185), bladder cancer (codes 188.x, 233.7, 236.7), renal cancer (code 189), testicular cancer (code 186.9), patients who have undergone RP (code 60.5), RC (code 57.71), PN (code 55.4), RN (code 55.5) and retroperitoneal lymphadenectomy (codes 40.2 and 40.5) between 2000 and 2020. Codes 17.42, 17.44, or 17.49 will be used to classify robotic procedures. We will examine yearly trends in the adoption rates of robotic procedures over time, defined as the percentage of procedures performed robotically.

\section{Systematic review and meta-analysis of the literature}

Patient and public involvement statement

Patients and the public were not involved in the development of the research questions, outcome measures and study design.

\section{Study design}

This study will be performed according to the Preferred Reporting Items for Systematic Review and Meta-Analysis Protocols 2015 guidelines $^{8}$ (online supplementary appendix A).

\section{Eligibility criteria}

A summary of the participants, interventions, comparators, outcomes and time and settings considered is provided, along with the type of studies included according to Population, Intervention, Comparators, Outcomes, Timing and Setting strategy. A detailed description is given in table 1.

\section{Types of studies to be included}

All available clinical prospective randomised and nonrandomised trials and retrospective comparative studies (cohort or case-control series) comparing ORP versus RRP, ORC versus RRC, OPN versus RPN, ORN versus 
Table 1 Description of the PICOTS strategy as applied to this study

\begin{tabular}{|c|c|}
\hline & Inclusion \\
\hline Population & $\begin{array}{l}\text { Age } \geq 18 \text { years } \\
\text { Diagnoses: urological neoplasia in adults: } \\
\text { Localised prostate cancer } \\
\text { Renal mass } \\
\text { Invasive bladder cancer } \\
\text { Testicular cancer }\end{array}$ \\
\hline Interventions & $\begin{array}{l}\text { RP (open vs robotic approach) } \\
\text { RC (open vs robotic approach) } \\
\text { PN (open vs robotic approach) } \\
\text { RN (open vs robotic approach) } \\
\text { RPLND (open vs robotic approach) }\end{array}$ \\
\hline Comparators & $\begin{array}{l}\text { Comparison between open and robotic } \\
\text { approaches in the treatment of urological } \\
\text { cancers included in the list above }\end{array}$ \\
\hline Outcomes & $\begin{array}{l}\text { Perioperative outcomes: } \\
\text { Operative time (min) } \\
\text { Estimated blood loss (mL) } \\
\text { Length of hospital stay (days) } \\
\text { Blood transfusion rate (\%) } \\
\text { Overall complication rate (\%) } \\
\text { Major and minor postoperative complication } \\
\text { rate }(\%) \\
\text { Early and late complication rate (\%) } \\
\text { Readmission rate (\%) } \\
\text { Oncological outcomes: } \\
\text { Positive margins } \\
\text { Lymph node counts } \\
\text { Cancer-specific survival } \\
\text { Overall survival } \\
\text { Functional outcomes: } \\
\text { Recurrence-free survival } \\
\text { Potency recovery rate }(\mathrm{n}) \\
\text { Continence recovery rate }(\mathrm{n}) \\
\text { Health-related quality-of-life } \\
\text { Henal function (eGFR change) } \\
\text { Hospital costs: } \\
\text { Operative costs } \\
\text { Non-operative costs }\end{array}$ \\
\hline
\end{tabular}

$\begin{array}{ll}\text { Type of studies } & \begin{array}{l}\text { All available clinical, prospective randomised } \\ \text { and non-randomised trials and retrospective } \\ \text { comparative studies (cohort or case-control } \\ \text { series) comparing RRP versus ORP, RRC } \\ \text { versus ORC, RPN versus OPN, RRN versus } \\ \text { ORN and RRPLND versus ORPLND were } \\ \text { included. Published between } 2000 \text { and } 2020 .\end{array} \\ \begin{array}{l}\text { Timing and } \\ \text { setting }\end{array} & \text { Any time point and setting }\end{array}$

eGFR, estimated glomerular rate; OPN, open partial nephrectomy; ORC, open radical cystectomy; ORN, open radical nephrectomy; ORP, open radical prostatectomy; ORPLND, open retroperitoneal lymph node dissection; PN, partial nephrectomy; RC, radical cystectomy; RN, radical nephrectomy; RP, radical prostatectomy; RPLND, retroperitoneal lymph node dissection; RPN, robotic partial nephrectomy; RRC, robotic radical cystectomy; RRN, robotic radical nephrectomy; RRP, robotic radical prostatectomy; RRPLND, robotic retroperitoneal lymph node dissection.

RRN and ORPLND versus RRPLND and published between 1st January 2000 and 10th January 2020 will be included. No language restrictions will be applied. Native speaking urologists will be involved in the translation of non-English publications.
Condition being studied

We will examine the literature of open versus robotic urological oncological surgery by assessing the most common oncological surgeries: RP for localised prostate cancer, RC for invasive bladder cancer, PN and RN for renal mass and RPLND for testis cancer.

\section{Type of participants/population}

We will include adult participants (age $\geq 18$ years), with diagnoses of urological neoplasia (localised prostate cancer, invasive bladder cancer, renal mass, testis cancer).

\section{Type of intervention and comparators}

We will evaluate the comparator intervention (open approach) versus the experimental intervention (robotic approach) separately for each surgical procedure: RP, RC, PN, RN and RPLND.

\section{Type of outcomes measures}

We will compare the following outcomes between open and robotic uro-oncological surgery:

1. Penetrance: US data for the number of surgeries performed annually for each procedure type (Premier Database).

2. Perioperative outcomes: operative time (min); estimated blood loss (mL); length of hospital stay (days); blood transfusion rate $(\%)$; complication rate $(\%)-$ overall, minor (Clavien-Dindo <3), major (ClavienDindo $\geq 3$ ), early (within 30 days), late (31-90 days); rate of specific complications (wound, anastmotic, cardiovascular, gastrointestinal, thromboembolic, infectious); and readmission rate $(\%)$.

3. Functional outcomes: at four time-points after surgery-early (at 3 months), intermediate (at 6 months), late (at 12 months) and overall (latest month) - assess erectile dysfunction rate $(\%)$ and incontinence rate (\%) after RP; and decline in estimated glomerular rate after $\mathrm{PN}$ and RN.

4. Oncological and survival outcomes: rate of positive surgical margins (\%); lymph node yield; recurrence rate $(\%)$; cancer-specific survival rate $(\%)$; and overall survival rate $(\%)$.

5. Hospital Costs: operative costs and non-operative costs.

\section{Search strategy for relevant studies}

The systematic review will be performed in accordance with the Cochrane Guidelines, ${ }^{9}$ the Preferred Reporting Items for Systematic Reviews and Meta-Analyses (PRISMA) ${ }^{10}$ and graded strength of evidence using the scheme recommended by Methods and Guide for effectiveness and Comparative Effectiveness Review of the Agency for Healthcare Research and Quality. ${ }^{11}$.

We will search Pubmed, Scopus and Web of Science databases for all published full-text English and nonEnglish language articles on the treatment of localised prostate, bladder, kidney and testis cancer, using the keywords 'radical prostatectomy' OR 'radical cystectomy' OR 'partial nephrectomy' OR 'radical nephrectomy' OR 'retroperitoneal lymph node dissection' published 
between 1st January 2000 and 10th January 2020. No language restriction will be applied (online supplementary appendix B). References will be manually reviewed to identify supplementary studies of interest.

We will exclude case series describing only one approach (robotic or open), studies not comparing open versus robotic approach, non-comparative series, multiinstitutional studies reporting overlapping data with series previously published, studies from the same institution with overlapping data, reviews, meta-analyses, surgical technique description papers, replies, commentaries and editorial comments, single case reports, papers about paediatric surgery, non-matching articles and studies not providing outcomes of interest,.

Multi-institutional studies that report data never published by participating single-centre studies will be considered. When an institution has published multiple papers with overlapping surgical periods, we will consider only the latest published paper. We will exclude studies analysing national databases because of the high risk of overlapping data. The words 'robot(ic)-assisted' and 'robotic' will be used interchangeably.

\section{Screening and data extraction}

Two paired investigators (GEC and ISG) will independently screen all articles focusing the research on open versus robotic surgery. All available clinical trials, prospective and retrospective studies (cohort or casecontrol series) comparing open versus robotic surgery will be included. Any disagreements about eligibility will be resolved by discussion between the paired investigators until a consensus is reached.

\section{Quality assessment}

All papers will be categorised according to the Oxford Level of Evidence Working Group 2011 levels of evidence for therapy studies. ${ }^{12}$ Two paired investigators (GEC and ISG) will independently weigh the risk of bias for all the studies according to the Cochrane Handbook for Systematic Reviews of Interventions for including nonrandomised studies. ${ }^{13}$ In consideration of the design of studies, we will likewise examine the risk of preassigned confounders identified as the possible predictors at the time of surgery. We will examine articles for specific data on baseline characteristics that may have an impact on outcomes of interest within their univariate analysis models.

\section{Statistical analysis}

Figure 1 summarises the steps of the present systematic review. A cumulative meta-analysis will be conducted using Review Manager V.5.3 (Cochrane Collaboration, Oxford, UK). Weighted mean difference will be used to compare continuous variables and odd ratio, and risk ratio and hazard ratio will be used to compare dichotomous variables, respectively. Baseline characteristics will be analysed in the same fashion.

All results will be reported with $95 \%$ CIs. Statistical heterogeneity between studies will be tested using the $\mathrm{I}^{2}$ statistic. ${ }^{9}$ Heterogeneity will be considered low, moderate

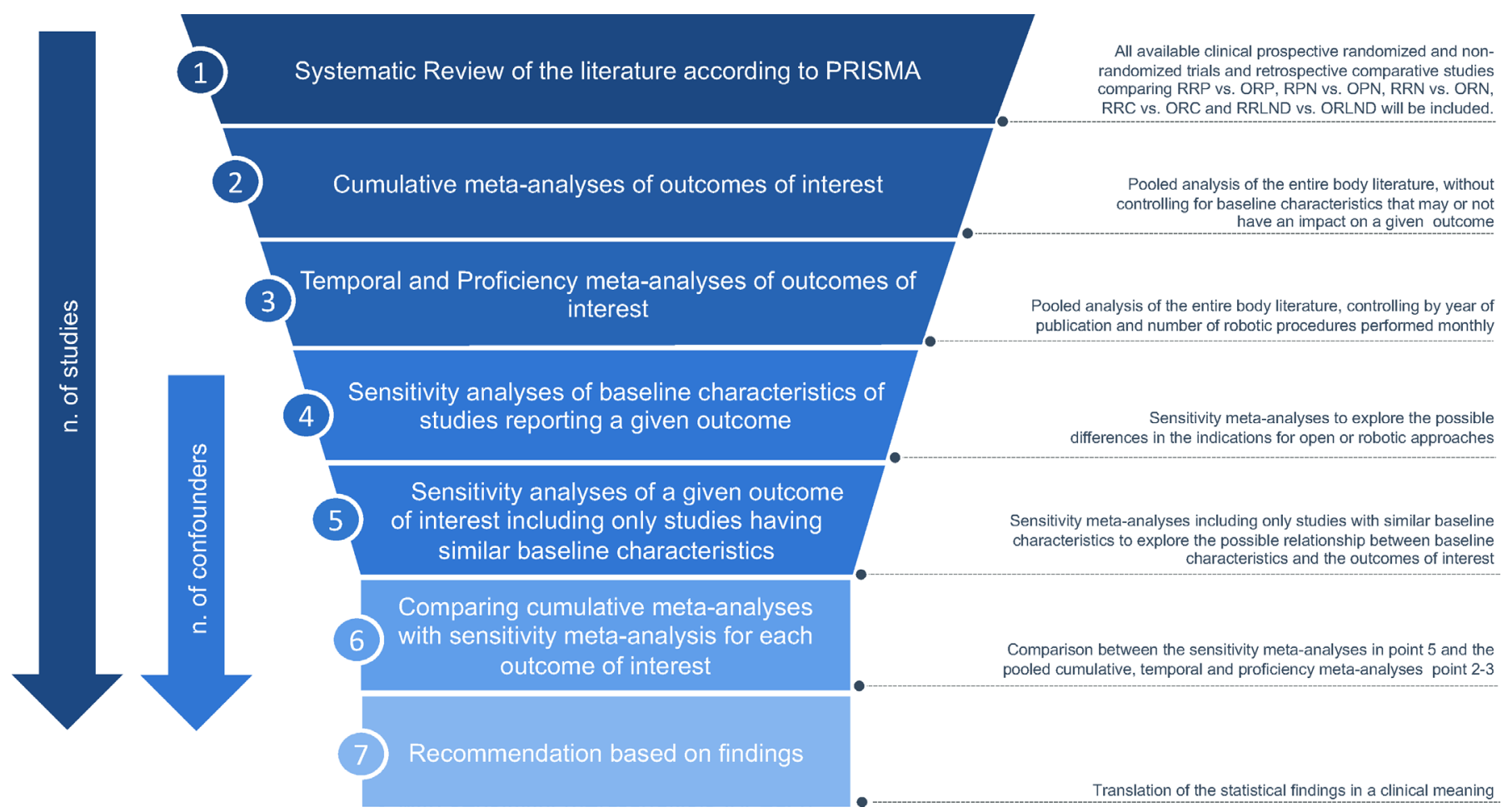

Figure 1 Study design flow chart. OPN, open partial nephrectomy; ORC, open radical cystectomy; ORN, open radical nephrectomy; ORP, open radical prostatectomy; ORPLND, open retroperitoneal lymph node dissection; RPN, robotic partial nephrectomy; RRC, robotic radical cystectomy; RRN, robotic radical nephrectomy; RRP, robotic radical prostatectomy; RRPLND, robotic retroperitoneal lymph node dissection. 
and high when the values are below $25 \%$, between $25 \%$ and $75 \%$ and above $75 \%$, respectively. ${ }^{9}{ }^{14}$

Random and fixed effects will be used in case of the presence or absence of heterogeneity, respectively. ${ }^{9} \mathrm{~A}$ two-sided $p$-value $<0.05$ will be considered statistically significant. Pooled analysis of continuous variables is possible only when data are presented as mean and SD. Since some studies may report continuous variables in 'median' and 'IQR' or ' $\mathrm{min} / \max$ ' range, we will use a validated mathematical method to estimate 'mean' and 'SD'. ${ }^{15}$ When available, we will use data reported in a matched-pair comparison manner.

The use of the pooled graphical effect of a meta-analysis that includes randomised controlled trials (RCTs) and non-RCTs allows assessment of the similarity between the studies. Forest plots allow the presentation of estimates and standard errors for each study. Graphical representation of pooled findings will be made according to the heterogeneity. ${ }^{9}$

\section{Assessment of publication bias}

Funnel plots will be visually inspected to assess both the degree of publication bias and its effect on the study findings. Egger's weighted regression will be used to test for publication bias, with $\mathrm{p}<0.1$ considered indicative of statistically significant publication bias. When asymmetry is found, extreme outliers (ie, small studies) will be removed from the funnel plot, Duval and Tweedie nonparametric 'trim and fill' analysis to formalise the use of funnel plot and recomputing the effect size to correct for publication bias. ${ }^{13}$ When necessary, to assess the risk of bias in the non-RCTs included in our meta-analysis, we will independently weigh the risk of bias for all studies according to the Cochrane Handbook for Systematic Reviews of Interventions for including non-randomised studies. ${ }^{9}$ In considering the design of studies, we will likewise examine the risk of preassigned confounders identified as possible predictors at the time of surgery. We will examine all publications for specific data on baseline characteristics that may impact outcomes of interest within their univariate analysis models.

\section{Sensitivity analyses}

\section{Temporal meta-analysis}

The chronologic time of publication may impact the reported outcomes of urological oncological surgery; in other words, earlier publications that reflect the 'discovery' era of a novel technology may demonstrate different/inferior outcomes than later publications, when the initial learning curve has been surmounted. As such, we will perform a temporal meta-analysis to evaluate the evolving impact of time of publication on reported outcomes of open and robotic uro-oncological surgery. First, a meta-regression model will be performed to assess the association between the year of publication and the outcomes of interest. Then, we will divide all available comparative studies into two equivalent temporal cohorts. Various outcomes of ORP versus RRP, ORC versus RRC,
OPN versus RPN, ORN versus RRN and ORPLND versus RRPLND will be compared between these two cohorts to assess the temporal impact of the robotic learning curve.

\section{Proficiency meta-analysis}

Similarly, surgical case volumes can impact the outcomes of urological oncological surgery; in other words, because of presumed differences in surgical 'proficiency', lowvolume centres may deliver inferior outcomes compared with high-volume centres. ${ }^{16}$ However, a clear definition of low-volume and high-volume centres is still lacking. To evaluate the impact of surgical volumes on the outcomes of interest, we will perform a 'proficiency' meta-analysiss. We will exclude all multi-centre studies, propensity score-matched studies and studies that do not report the surgical period. The mean number of robotic procedures performed per month will be calculated by dividing the total number of robotic procedures reported by the number of months in the study period. First, a metaregression model will be performed to assess the association between the number of robotic procedures/month and the outcomes of interest. Next, we will calculate the mean number of procedures/month to determine the cut-off to distinguish between low-volume (number of robotic procedures/month below the cut-off value) versus high volume (number of robotic procedures/ month above the cut-off value) centres. Various outcomes of ORP versus RRP, ORC versus RRC, OPN versus RPN, ORN versus RRN and ORPLND versus RRPLND will be compared between low-volume and high-volume centres. Since data on single-surgeon experience in studies comparing open versus robotic uro-oncology surgery are sparse, those cut-offs will be considered as a surrogate to evaluate the impact of surgical skill proficiency on perioperative, functional and oncological outcomes.

\section{Assessment of baseline characteristics}

Differences in baseline characteristics between cohorts may be present in the studies included in a meta-analysis. Ignoring such substantial variability in one or more baseline characteristics may lead to misleading conclusions, which can jeopardise the overall applicability of the pooled estimates. ${ }^{17}$ Therefore, comparability of baseline patient characteristics between the two cohorts is crucial for minimising the impact of heterogeneity on study outcomes.

To account for the impact of baseline characteristics on a given outcome and explore the possible relationship between baseline characteristics and outcomes of interest, we will perform a pooled analysis of the baseline characteristics (table 2). Then, we will perform a sensitivity analysis of the pooled estimate of the baseline characteristics reported by the studies reporting a given outcome to assess differences between the two cohorts. Sensitivity analyses will be performed plotting only papers reporting comparable baseline characteristics estimates (weighted mean difference for continues variable and OR for dichotomous variable) defined as estimates with 


\begin{tabular}{|c|c|}
\hline Surgical procedure & Baseline characteristics \\
\hline \multirow{12}{*}{$\begin{array}{l}\text { RP } \\
\text { (ORP vs RRP) }\end{array}$} & Age, years \\
\hline & $\mathrm{BMI}, \mathrm{kg} / \mathrm{m}^{2}$ \\
\hline & ASA score \\
\hline & $\mathrm{PSA}, \mathrm{ng} / \mathrm{mL}$ \\
\hline & Clinical GS $\leq 6, \%$ \\
\hline & Clinical GS=7, \% \\
\hline & Clinical GS $\geq 8, \%$ \\
\hline & Pathological GS $\leq 6, \%$ \\
\hline & Pathological GS=7, \% \\
\hline & Pathological GS $\geq 8, \%$ \\
\hline & pT $\geq 3, \%$ \\
\hline & $\mathrm{pN} \geq 1, \%$ \\
\hline \multirow{9}{*}{$\begin{array}{l}\text { RC } \\
\text { (ORC vs RRC) }\end{array}$} & Age, years \\
\hline & $\mathrm{BMl}, \mathrm{kg} / \mathrm{m}^{2}$ \\
\hline & ASA score \\
\hline & Male, \% \\
\hline & Female, \% \\
\hline & $\mathrm{NACH}, \%$ \\
\hline & pT $\geq 3, \%$ \\
\hline & $\mathrm{pN} \geq 1, \%$ \\
\hline & number of nodes removed, mean \\
\hline \multirow{12}{*}{$\begin{array}{l}\text { PN (OPN vs RPN) and RN (ORN vs } \\
\text { RRN) }\end{array}$} & Age, years \\
\hline & $\mathrm{BMI}, \mathrm{kg} / \mathrm{m}^{2}$ \\
\hline & ASA score, $\%$ \\
\hline & Male, \% \\
\hline & Preoperative eGFR \\
\hline & Left/right side, \% \\
\hline & Tumour size, $\mathrm{cm}$ \\
\hline & Renal score \\
\hline & Renal score $\leq 6, \%$ \\
\hline & Renal score $7-10, \%$ \\
\hline & Renal score $11-12, \%$ \\
\hline & pT $\geq 1 \mathrm{~b}, \%$ \\
\hline \multirow{10}{*}{$\begin{array}{l}\text { RPLND } \\
\text { (ORPLND vs RRPLND) }\end{array}$} & Age, years \\
\hline & $\mathrm{BMI}, \mathrm{kg} / \mathrm{m}^{2}$ \\
\hline & ASA score \\
\hline & Primary laterality left/right side, $\%$ \\
\hline & Preoperative AFP (ng/mL) \\
\hline & Preoperative hCG (mlU/mL) \\
\hline & Lympho vascular invasion, \% \\
\hline & $\mathrm{pT} \geq 2, \%$ \\
\hline & $\mathrm{pN} \geq 1, \%$ \\
\hline & number of nodes removed, mean \\
\hline
\end{tabular}

AFP, Alpha Fetoprotein; ASA, American Society of Anaesthesiologists; BMI, body mass index; eGFR, estimated glomerular rate; GS, Gleason Score; hCG, human chorionic gonadotropin; $\mathrm{NACH}$, neoadjuvant chemotherapy; OPN, open partial nephrectomy; ORC, open radical cystectomy; ORN, open radical nephrectomy; ORP, open radical prostatectomy; ORPLND, open retroperitoneal lymph node dissection; $\mathrm{pN}$, pathologica node stage; PN, partial nephrectomy; PSA, prostate specific antigen ; pT, pathological tumor stage; RC, radical cystectomy; RN, radical nephrectomy; RP, radical prostatectomy; RPLND, retroperitoneal lymph node dissection; RPN, robotic partial nephrectomy; RRC, robotic radical cystectomy; RRN, robotic radical nephrectomy; RRP, robotic radical prostatectomy; RRPLND, robotic retroperitoneal lymph node dissection.
$95 \%$ CI for effect including the null value (such as an OR of 1.0 or a risk difference of 0$).^{13}$

\section{Risk of incontinence and erectile dysfunction after RP}

For the analysis of continence and potency recovery rates following RP (open vs robotic), we will assess the relative risk of incontinence and erectile dysfunction, respectively. We will consider as 'total' the number of men reporting to be continent or potent before surgery or, if not specified, the total number of men evaluated at last follow-up. We will consider as 'events' the total number of men who report having incontinence or erectile dysfunction after surgery. This number will be calculated by the difference $(\Delta)$ between the total of the "continent or potent patients following surgery' and the 'total' (the number of patients who are continent or potent before surgery or, if not specified, the total number of patients evaluated at last follow-up). Inconsistency in the definition will be reported.

\section{Cost analyses}

We will evaluate the costs of open versus robotic urooncological surgery. We will select only comparative studies (open vs robotic) reporting operative and nonoperative costs; studies reporting charges, modelling and analyses of national databases will be excluded. We will include the following items in operative costs: (1) labour (professional fees); (2) surgical equipment; (3) robot-related costs (capital, maintenance); and (4) anaesthesia supply/technician labour cost. We will include the following items in non-operative costs: (1) postanaesthesia care; (2) professional fees; (3) hospital stay costs (room/day, nursing costs); (4) drugs and supplies; (5) blood transfusion costs; and (6) technical services (laboratory, radiology). Difference $(\Delta)$ in overall operative cost will be calculated as overall robotic operative cost minus overall open operative cost. Difference $(\Delta)$ in overall non-operative cost will be calculated as overall robotic non-operative cost minus overall open non-operative cost. Percentages indicating the overall total cost differential of robotic vis-a-vis open surgery will be considered. We will convert all currencies to the 2020 exchange rate using the Consumer Price Index (CPI) inflation calculator (https://www.bls.gov/data/inflation_calculator. $\mathrm{htm})$. Foreign currency will first be converted to dollars using historical exchange rates at the year of publication and then adjusted to the 2020 exchange rate using the CPI inflation calculator.

\section{Grade of the evidence}

The quality of scientific evidence and outcomes will be assessed using the Grading of Recommendations Assessment, Development and Evaluation (GRADE) approach. GRADE offers a specific definition of the quality of evidence that is different in the context of making recommendations and in the context of summarising the findings of a systematic review. ${ }^{18} 19$ The GRADE methodology involves rating evidence for a given outcome 
by upgrading or downgrading the evidence. Indications for upgrading the quality of evidence include having a large effect size and dose-response gradient. Indications for downgrading the quality of evidence include serious risk of bias, serious inconsistency between studies, serious indirectness, serious imprecision and likely publication bias. ${ }^{18}$ Summary of Findings (SoF) tables will provide a summary of findings for each of the included outcomes and the quality of evidence rating for each outcome. ${ }^{18}$ The format of the SoF will include:

1. A list of the outcomes of interest.

2. The assumed risk; a measure of the typical burden of the outcomes, that is, illustrative risk or also called baseline risk, baseline score or control group risk.

3. The corresponding risk; a measure of the burden of the outcomes after the intervention is applied, that is, the risk of an outcome in treated/exposed people based on the relative magnitude of an effect and assumed (baseline) risk.

4. The relative effect; for dichotomous outcomes, the table will provide risk ratio, OR or HR.

5 . The number of participants, the number of studies and their designs

6. Rating of the overall quality of evidence for each outcome.

\section{Presentation of results and reporting}

The PRISMA guidelines ${ }^{10}$ will be used and the checklist will accompany the publication. Quantitative data will be summarised and presented in tables and as forest plots where necessary. ${ }^{9}$

\section{Potential amendments}

We do not anticipate any amendments to the current protocol. However, should an amendment be necessary, it will be notified, registered and reported.

\section{Ethics and Dissemnination}

No ethics clearance is necessary, as no primary data will be collected. Results will be published in a peer-reviewed journal. These results will likely help inform directions and design of future studies.

\section{IMPLICATIONS OF THE REVIEW}

We believe this aggregate information will be of interest and practical use to the general medical community at large, who need to be aware of contemporary trends in urological oncological surgery, thereby to better advise patients seeking care for urological cancers.

Contributors ISG and GEC conceptualised and designed the protocol, drafted the initial manuscript and reviewed the manuscript. ISG and GEC defined the concepts and search items, data extraction process as well as methodological appraisal of the studies. GEC, KG and ISG planned the data extraction and statistical analysis.
ISG and GEC provided critical insights. All authors have approved and contributed to the final written manuscript.

Funding The authors have not declared a specific grant for this research from any funding agency in the public, commercial or not-for-profit sectors.

Competing interests None declared.

Patient consent for publication Not required.

Provenance and peer review Not commissioned; externally peer reviewed.

Open access This is an open access article distributed in accordance with the Creative Commons Attribution Non Commercial (CC BY-NC 4.0) license, which permits others to distribute, remix, adapt, build upon this work non-commercially, and license their derivative works on different terms, provided the original work is properly cited, appropriate credit is given, any changes made indicated, and the use is non-commercial. See: http://creativecommons.org/licenses/by-nc/4.0/.

ORCID iD

Giovanni E Cacciamani http://orcid.org/0000-0002-8892-5539

\section{REFERENCES}

1 Data I.s, 2017. Available: https://www.youtube.com/watch?v= 1CHUIrfM9C4

2 Childers CP, Maggard-Gibbons M. Estimation of the acquisition and operating costs for robotic surgery. JAMA 2018;320:835-6.

3 Leow JJ, Cole AP, Seisen T, et al. Variations in the costs of radical cystectomy for bladder cancer in the USA. Eur Urol 2018;73:374-82.

4 Leow JJ, Chang SL, Meyer CP, et al. Robot-assisted versus open radical prostatectomy: a contemporary analysis of an all-payer discharge database. Eur Urol 2016;70:837-45.

5 Cheung H, Wang Y, Chang SL, et al. Adoption of robot-assisted partial nephrectomies: a population-based analysis of U.S. surgeons from 2004 to 2013. J Endourol 2017;31:886-92.

6 Lindenauer PK, Pekow P, Wang K, et al. Perioperative beta-blocker therapy and mortality after major noncardiac surgery. $N$ Engl $J$ Med 2005;353:349-61.

7 Wright JD, Ananth CV, Lewin SN, et al. Robotically assisted vs laparoscopic hysterectomy among women with benign gynecologic disease. JAMA 2013;309:689-98.

8 Moher D, Shamseer L, Clarke M, et al. Preferred reporting items for systematic review and meta-analysis protocols (PRISMA-P) 2015 statement. Syst Rev 2015;4:1.

9 Higgins J GS. Cochrane Handbook for systematic reviews of interventions version 6.0; 2019.

10 Moher D, Liberati A, Tetzlaff J, et al. Preferred reporting items for systematic reviews and meta-analyses: the PRISMA statement. Int J Surg 2010;8:336-41.

11 Quality, A.A.f.H.R.a. Methods guide for effectiveness and comparative effectiveness reviews, 2015. Available: http:// effectivehealthcare.ahrq.gov/index.cfm/search-for-guides-reviewsand-reports/?pageaction=displayproduct\&productid $=318$

12 Howick J, Glasziou P, Greenhalgh T, et al. The Oxford 2011 levels of evidence, Oxford centre for evidence-based medicine, 2011. Available: http://www.cebm.net/index.aspx?o=5653

13 Higgins JPT. Cochrane Handbook for systematic reviews of interventions version 5.1.0, 2011. Available: http://handbook. cochrane.org [Accessed Mar 2011].

14 Higgins JPT, Thompson SG. Quantifying heterogeneity in a metaanalysis. Stat Med 2002;21:1539-58.

15 Wan X, Wang W, Liu J, et al. Estimating the sample mean and standard deviation from the sample size, median, range and/or interquartile range. BMC Med Res Methodol 2014;14:135.

16 Bach PB, Cramer LD, Schrag D, et al. The influence of hospital volume on survival after resection for lung cancer. N Engl J Med 2001;345:181-8.

17 Chaimani A. Accounting for baseline differences in meta-analysis. Evid Based Ment Health 2015;18:23-6.

18 GRADEpro. Available: https://gdt.gradepro.org/app/handbook/ handbook.html\#h.wlsfq2lmj0gb

19 Guyatt GH, Oxman AD, Vist GE, et al. Grade: an emerging consensus on rating quality of evidence and strength of recommendations. BMJ 2008;336:924-6. 